Kinetic Interaction of Microparticles in Solution

T. Arndt

Bioscientia Institut für Medizinische Diagnostik GmbH, Ingelheim, Deutschland

\section{Synonym(e) KIMS}

Englischer Begriff kinetic interaction of microparticles in solution

Definition Sonderform des Immunoassays ( $\triangleright$ Immunoassay) und hier des latexverstärkten Immunoassays.

Beschreibung Im Reaktionsansatz konkurrieren Antigenbeschichtete Latexpartikel mit Antigenen der Patientenprobe um eine limitierte Anzahl von gegen das Antigen gerichteten Antikörpern. Sind in der Patientenprobe keine oder wenig Antigene vorhanden, binden die Antikörper vorrangig an die auf den Latexpartikeln fixierten Antigene und vernetzen dabei mit ihren beiden Bindungsstellen einzelne Latexpartikel zu einem Netzwerk. Im Ergebnis wird der Reaktionsansatz trüb. Enthält die Patientenprobe Antigenmoleküle, bindet ein Teil dieser Antigene an die limitiert verfügbaren Antikörper. In der Folge stehen weniger Antikörper zur Vernetzung der Latexpartikel zur Verfügung. Die Trübung des Reaktionsansatzes fällt dann schwächer aus. Zwischen der Trübung des Reaktionsansatzes und der einhergehenden Abschwächung der Lichtintensität vor Eintritt in den Reaktionsansatz und nach dessen Passage besteht ein mathematischer Zusammenhang ( $\triangleright$ Lambert-Beer-Gesetz). Über eine geeignete Kalibrationsfunktion ist aus der Trübung (oder der Extinktion) des Reaktionsansatzes auf die Konzentration des zu bestimmenden Antigens in der Patientenprobe zu schließen. Das Testprinzip findet $u$. a. Anwendung im $>$ Drogenscreening.

\section{Literatur}

Armbruster DA, Schwarzhoff RH, Hubster EC, Liserio MK (1993) Enzyme immunoassay, kinetic microparticle immunoassay, radioimmunoassay, and fluorescence polarization immunoassay compared for drugs-ofabuse screening. Clin Chem 39:2137-2146 